\title{
L'impact de la sélection et du profil académique sur la performance cognitive d'étudiants universitaires
}

\author{
DANIEL PELLETIER ${ }^{\prime}$, SERGE LARIVÉE $^{2}$, SYLVAIN COUTU $^{1}$, \\ SOPHIE PARENT ${ }^{2}$
}

\section{RÉSUMÉ}

L'évaluation d'adultes à l'aide d'épreuves piagétiennes indique clairement que la maîtrise des opérations formelles est beaucoup moins généralisée que ce que la théorie laisse supposer. Chez les étudiants universitaires, ce décalage entre la performance et la compétence attendue est souvent attribué au manque de familiarité avec le contenu. Dans le cadre de cette recherche, une autre variable rarement prise en considération a été retenue: la sélection à l'entrée des candidats admis dans les divers programmes. Un échantillon de 740 étudiants universitaires provenant de 12 concentrations différentes a été évalué à l'aide du Piagetian Logical Operations Test. Les données recueillies mettent en évidence une performance globale supérieure des étudiants inscrits en sciences pures et appliquées. Cependant, les étudiants inscrits dans les programmes plus sélectifs de sciences humaines et sociales ont des résultats comparables à ceux des étudiants provenant de programmes à haute teneur mathématique et/ou scientifique, mais n'ayant pas fait l'objet d' une sélection serrée. L'impact de la sélection et $d u$ regroupement des sujets est discuté. L'interprétation traditionnelle des différences de performance en termes de déficit opératoire est critiquée, et des retombées possibles sur l'enseignement universitaire sont présentées.

Les auteurs tiennent à remercier les personnes suivantes, qui ont participé à cette recherche : Maryse Beaudoin, Martine Beaurivage, Chantal Bégin, Nathalie Bilodeau, Nancy Boislard, Nathalie Deschènes, France Dussault, Linda Guimond. Sophie Jobin, Elyse Lemyre, Monique Martineau. Loraine Nadeau, Sylvie Normandeau, Daniel Paradis, Martin Pigeon, Roch Riendeau, Sylvain Villemaire.

\section{${ }^{1}$ Université du Québec à Hull}

${ }^{2}$ Ecole de Psycho-Éducation, Université de Montréal

"L'utilisation exclusive du masculin pour désigner les étudiantes et les étudiants ne sert qu'à alléger le texte. 


\begin{abstract}
The assessment of adults by means of Piagetian tests clearly shows that the mastery of formal operations is much less widespread than the theory had led us to believe. Among university students, the discrepancy between performance and expected skills was often attributed to their not being familiar with the contents of the tasks. In this research, another variable which has rarely been taken into account was considered: the selection of candidates for entry into various programs. A sampling of 740 university students majoring in 12 different fields were assessed by means of the Piagetian Logical Operations Test. The data thus collected clearly showed a higher overall performance of students in pure and applied sciences. However, students in more selective social and human science programs produced results comparable with those of students in programs having a high mathematical and/or scientific content, but who were not subjected to a careful selection process. The impact of the selection and grouping of the subjects is discussed. The traditional interpretation of variations in performance in terms of operational deficits is critically examined, and the possible effects on university teaching are presented.
\end{abstract}

Dans le cadre de la théorie piagétienne, le stade des opérations formelles habituellement atteint à l'adolescence est considéré comme la dernière étape du développement cognitif (Inhelder et Piaget, 1955; Piaget, 1970). Toutes les recensions des écrits concernant l'acquisition de la pensée formelle parviennent cependant au même constat: rarement plus de $50 \%$ des divers échantillons d'adolescents et de jeunes adultes sont évalués au niveau opératoire formel (Alcock et Moore, 1985; De Garcer, Gabel et Staver, 1978; Higelé, 1978; Larivée et Cormier, 1982; Larivée et Normandeau 1985; Larivée et Roulin, 1985; McKinnon et Renner, 1971).

Si cette faible performance aux épreuves opératoires classiques peut s'expliquer partiellement chez les adolescents et les adultes peu scolarisés par un manque d'opportunités de développer et d'exercer leurs schèmes formels, on devrait s'attendre à une bonne performance chez les étudiants qui accèdent au niveau universitaire. En effet, les programmes universitaires exigent habituellement un haut niveau d'abstraction caractéristique de la pensée formelle (raisonnement hypothético-déductif, logique propositionnelle, ouverture sur les possibles, etc.). Qui plus est, la scolarité pré-universitaire comprend certains programmes dont l'assimilation des contenus (v.g. mathématiques, physique, chimie) nécessite l'utilisation des schèmes formels (combinatoire, contrôle des variables, corrélation, probabilité, proportion).

Force est cependant de constater que les données empiriques disponibles concernant le niveau de développement cognitif des étudiants universitaires ne rencontrent pas les prévisions théoriques, non seulement en ce qui a trait à la maîtrise des schèmes formels, mais aussi en ce qui concerne la prédiction de la 
performance scolaire à l'université à partir de la réussite aux épreuves formelles (Albanese et Brooks, 1976; Commons, Miller et Kuhn, 1982; El-Gosbi, 1982). En plus des pourcentages de réussite relativement faibles, on observe une assez grande variabilité des résultats. Si celle-ci peut être attribuée au type de passation des épreuves, aux schèmes étudiés ou aux critères d'analyse utilisés (Larivée, Pelletier et Gagnon, 1986), des différences de performance sont aussi observées en fonction des programmes dans lesquels les étudiants sont inscrits. Ainsi, dans la majorité des cas, les étudiants inscrits aux programmes à haute teneur scientifique et/ou mathématique présentent une performance supérieure à celle de leurs collègues inscrits aux programmes de sciences humaines et sociales (Dunbar et Taylor, 1983; Good, 1983; Larivée et Normandeau, 1984; Parete, 1978; Torkia-Lagacé, 1981; White et Ferstenberg, 1978).

À la limite, un tel résultat n'est guère surprenant. Même si l'indépendance de la forme et du contenu est censée caractériser le raisonnement opératoire formel, l'atteinte de ce niveau de raisonnement est fonction des intérêts et de la spécialisation professionnelle (Bart, 1971; Piaget, 1970). Dans cette perspective, les épreuves piagétiennes classiques utilisées pour mesurer l'atteinte du stade formel favorisent, en raison de leur nature logico-mathématique, une meilleure performance des individus qui sont familiers avec les contenus à caractère scientifique.

Les efforts entrepris en vue d'adapter le contenu d'épreuves formelles au champ d'intérêt et au domaine de spécialisation des sujets évalués ont permis de spécifier davantage en quoi la familiarité avec le contenu affectait la performance (Enwieme, 1976; Kuhn et Brannock, 1977; Neimark, 1978, 1979; Pulos et Linn, 1981). Ainsi, DeLisi et Staudt (1980) ont utilisé trois épreuves formelles différentes dont le contenu correspondait à un domaine de spécialisation universitaire : les oscillations du pendule, l'analyse stylistique et la socialisation politique. Trois groupes de sujets inscrits en physique, en langues et en sciences politiques furent évalués. Pour chaque programme, le nombre de sujets évalué au niveau formel était supérieur à $80 \%$ à l'épreuve qui correspondait au domaine de spécialisation, alors qu'il variait de 40 à $60 \%$ aux épreuves dont le contenu n'était pas familier.

Bien que la familiarité avec le contenu et la modalité d'administration puissent expliquer en partie les nombreux décalages compétence/performance relevés dans la littérature, il ne faut pas pour autant conclure trop hâtivement que les épreuves opératoires classiques discriminent de façon si évidente les étudiants en fonction de leur seule exposition au contenu scientifique; la plupart des recherches recensées font en effet plus ou moins abstraction des différences individuelles en tant que variable intermédiaire médiatisant l'impact du contenu sur la performance. À cet égard, la rigueur de la sélection à l'entrée des sujets étudiés varie beaucoup d'un programme à l'autre en raison du nombre variable de demandes d'admission, et pourrait générer des différences importantes au plan des capacités et des compétences des étudiants sélectionnés. Comme la sélection est la plupart du temps effectuée à partir des résultats scolaires aux études pré- 


\section{TABLEAU 1}

Description de l'échantillon en fonction des programmes, du sexe, de l'âge, de la sélection et du profil.

\begin{tabular}{|c|c|c|c|c|c|c|c|}
\hline \multirow[t]{2}{*}{ PROGRAMMES } & \multicolumn{2}{|c|}{ SEXE } & \multirow{2}{*}{$\begin{array}{l}\text { TOTAL } \\
\mathrm{N}\end{array}$} & \multicolumn{2}{|c|}{ AGE } & \multirow[t]{2}{*}{$\%$ DE REFUS $b$} & \multirow{2}{*}{$\begin{array}{l}\text { PROFIL } \\
\text { ACADEMIQUE }\end{array}$} \\
\hline & $M$ & $F$ & & MOY. & E.T. & & \\
\hline B.A.S. ${ }^{a}$ & 15 & 82 & 97 & 20,9 & 2,2 & 8,3 & III \\
\hline PSYCHOLOGIE & 2 & 22 & 24 & 27,0 & 6,08 & 65,1 & III \\
\hline BIOLOGIE & 39 & 35 & 74 & 20,6 & 2,2 & 2,0 & 1 \\
\hline DROIT & 19 & 23 & 42 & 21,1 & 2,7 & 64,1 & III \\
\hline GÉNIE & 35 & 8 & 43 & 19,9 & 0,8 & 57,3 & 1 \\
\hline PHILOSOPHIE & 22 & 8 & 30 & 23,1 & 3.6 & 2,0 & III \\
\hline INFORMATKQUE & 38 & 30 & 68 & 20,9 & 2,2 & 14,6 & 11 \\
\hline B.E.P.P. ${ }^{a}$ & 8 & 60 & 68 & 20,9 & 2.9 & 42,2 & 111 \\
\hline AOMINISTRATION & 57 & 52 & 109 & 20,5 & 2,5 & 42,9 & 11 \\
\hline MÉDECNE & 6 & 18 & 24 & 20,0 & 1.38 & 86,4 & 1 \\
\hline SC.INF. ${ }^{a}$ & 12 & 48 & 60 & 27.6 & 6,26 & 1,0 & III \\
\hline PS.ED. ${ }^{a}$ & 22 & 79 & 101 & 21,5 & 3,02 & 49,8 & 111 \\
\hline TOTAL & 275 & 465 & 740 & 21,66 & 3,08 & & \\
\hline
\end{tabular}

a B.A.S.: Baccalauréat en adaptation scolaire

B.E.P.P.: Baccalauréat en enseignement pré-scolaire et primaire

SC.INF:: Sciences infirmières

PS.ED.: Psycho-éducation

b Pourcentage de candidats refusés à la sélection initiale.

c 1: Diplôme d'études collégiales avec cours de mathématiques, physique et chimie

11: Diplôme d'études collégiales avec cours de mathématique

III: Diplôme d'études collégiales sans cours de sciences

universitaires et que ces dernières mettent à contribution les aptitudes intellectuelles générales (Kornbrot, 1987), il est plus que probable que les étudiants inscrits dans des programmes universitaires très sélectifs se démarqueront de ceux n'ayant pas été soumis à un tamisage minimal et ce indépendamment du contenu.

L'impact de la sélection sur la performance cognitive a déjà été démontré chez les pré-adolescents (Karplus, Karplus, Formisano et Paulsen, 1977), mais il est remarquablement absent de la problématique exposée ici. La présente recherche a pour but de combler partiellement cette lacune en étudiant la performance cognitive d'étudiants universitaires en prenant en considération le profil académique et la sélection à l'entrée des programmes dans lesquels ils sont inscrits. 


\section{MÉTHODOLOGIE}

\section{Sujets}

Les 740 sujets de l'étude sont des étudiants de première année inscrits dans un programme menant à une diplomation universitaire de premier cycle au Québec. Le tableau 1 présente la distribution des sujets en fonction des programmes d'appartenance, ainsi que les données relatives au profil académique et à la sélection des candidats à l'entrée obtenues du registrariat de l'université.

Les sujets provenant des divers programmes sont regroupés selon deux critères (voir le tableau 2). Le premier critère de regroupement utilisé est la sélection à l'entrée, qui comprend deux niveaux: un pourcentage de candidats refusés à l'entrée inférieur à $15 \%$ ou supérieur à $42 \%$. Le deuxième critère utilisé est le profil académique, c'est-à-dire les études antérieures requises pour être admissible aux divers programmes, ainsi que le contenu spécifique auquel les étudiants sont confrontés lors de leur première année universitaire. Cette variable comprend trois catégories : le profil I, caractérisé par la présence de cours de physique, de chimie, de biologie et de mathématiques dans la structure d'accueil et dans le programme actuel; le profil II, axé principalement sur les mathématiques; et le profil III, plus typique des programmes de sciences humaines et sociales, où les cours de sciences et de mathématiques sont à toutes fins pratiques absents.

La familiarité avec des contenus scientifiques et logico-mathématiques similaires à ceux des épreuves piagétiennes classiques est donc maximale pour les étudiants du profil I, intermédiaire pour ceux du profil II, et minimale pour ceux du profil III.

Les six cellules ainsi créées sont constituées d'étudiants provenant soit d'un seul programme (groupes $B, C$, et $D$ ), soit de programmes divers qui relèvent du même profil et du même niveau de sélection (groupes A, E, et F).

\section{Instrument}

La performance cognitive, est mesurée à l'aide d'une mesure collective de type papier/crayon, le P.L.O.T. (Piagetian Logical Operations Test; Staver, 1982).

Le P.L.O.T. est un test collectif de type papier-crayon, à choix multiples, accompagné d'une démonstration audio-visuelle illustrant de façon concrète le matériel et les données de chaque épreuve.

Il comprend cinq épreuves et mesure quatre schèmes: la dissociation poids/ volume (conservation), la flexibilité des tiges (contrôle des variables), la combinaison des corps chimiques (combinatoire), Mr. Tall Mr. Short et la balance (proportions). L'épreuve de dissociation du poids et du volume n'a pas été retenue ici en raison de contraintes horaires. Les résultats aux deux épreuves relevant du schème des proportions sont agrégés en raison de leur isomorphisme cognitif et du petit nombre d'items.

Les quatre épreuves comprennent un total de 51 items eux-mêmes répartis en trois types: des items d'information, vérifiant la compréhension des consignes, 


\section{Tableau 2}

Regroupements de programmes en fonction du pourcentage de refus et du profil académique.

\begin{tabular}{|c|c|c|}
\hline $\begin{array}{l}\text { Profil } \\
\text { académique }\end{array}$ & \multicolumn{2}{|c|}{ \% de candidats refusés à la sélection } \\
\hline Profil $1^{*}$ & $\begin{array}{l}\text { GROUPEA } \\
\text { Médecine } \\
\text { Génie } \\
N=67\end{array}$ & $\begin{array}{l}\text { GROUPEB } \\
\text { Biologie } \\
N=74\end{array}$ \\
\hline Profil 11 & $\begin{array}{l}\text { GROUPEC } \\
\text { Administration } \\
N=109\end{array}$ & $\begin{array}{l}\text { GROUPED } \\
\text { Informatique } \\
\mathrm{N}=68\end{array}$ \\
\hline Profil III & $\begin{array}{l}\text { GROUPEE } \\
\text { Psychologie } \\
\text { Droit } \\
\text { Psycho-éducation } \\
\text { B.E.P.P. } \\
N=235\end{array}$ & $\begin{array}{l}\text { GROUPEF } \\
\text { Philosophie } \\
\text { Sciences infirmières } \\
\text { B.A.S. } \\
N=187\end{array}$ \\
\hline
\end{tabular}

a 1: sciences pures et appliquées

11: mathématiques

III: sciences sociales et humaines

des items de décision qui requièrent un choix cognitif et des items de justification qui motivent la décision prise. Chaque question est accompagnée de quatre choix de réponse, dont une seule indique l'utilisation de schèmes formels. Le nombre de questions réussies aux items de décision et de justification est comptabilisé et donne un score pour chaque schème ainsi qu'un score global.

L'ampleur de l'échantillon nécessitait l'utilisation d'une épreuve collective, et le P.L.O.T. présente le double avantage de pouvoir être administré en grand groupe, tout en retenant les qualités essentielles associées à la méthode clinique traditionnelle, telles que la présence d'une illustration concrète de l'épreuve, la possibilité de vérifier la compréhension des consignes ainsi que l'adéquacité des 
justifications des réponses fournies par le biais de questions centrées sur la maitrise des données du problème ainsi que sur le raisonnement utilisé (Pelletier, Larivée et Gagnon, 1985).

La validité de construit du P.L.O.T. a été évaluée par trois séries d'analyses différentes: la matrice d'intercorrélations de Campbell et Fiske, l'analyse factorielle avec rotation Varimax et le coefficient Alpha (Staver et Gabel, 1979). L'analyse des corrélations indique une convergence satisfaisante entre la performance globale au P.L.O.T. et les résultats obtenus par la méthode clinique $(\mathrm{r}=.59, \mathrm{p}<.05)$, alors que l'analyse factorielle révèle que ces deux mesures sont saturées de facteurs différents : le P.L.O.T. obtient des coefficients de charge plus élevés au facteur I (intelligence générale) et au facteur III (développement cognitif mesuré par le P.L.O.T.), alors que la méthode clinique est surtout saturée du facteur II (développement cognitif mesuré par la méthode clinique). Le coefficient Alpha obtenu pour le score total au P.L.O.T. est de 0,85 . La validité de construit semble donc adéquate pour les fins de cette recherche, malgré les différences relevées entre ce test collectif et la méthode clinique. Au demeurant, cette incohérence apparente met bien en évidence en quoi le type de passation influence la performance. Dans cette perspective, une épreuve collective dont le contenu est typiquement piagétien et qui de plus comprend une composante importante d'intelligence générale semble toute indiquée.

\section{Procédure}

Chaque groupe d'étudiants a été évalué en classe. La distribution du test était suivie de la démonstration audio-visuelle illustrant le contenu de chaque épreuve. Par la suite, chacun répondait au test individuellement. Aucun temps limite n'était fixé, mais la session d'évaluation se terminait généralement après quarante-cinq minutes. Toutes les évaluations furent réalisées à la fin de la première année universitaire pour que l'exposition au contenu spécifique de chaque programme soit équivalente d'un groupe à l'autre.

\section{Résultats}

Les résultats obtenus par les étudiants de chacun des programmes aux items d'information et aux épreuves du contrôle des variables, de la combinatoire, des proportions et à l'ensemble des épreuves sont présentés au tableau 3.

La réussite des items d'information est généralisée, alors que le pourcentage de réponses formelles à chaque épreuve et au total est plus faible mais demeure tout de même supérieur à $50 \%$ dans tous les cas, sauf pour les étudiants du programme de philosophie, au schème des proportions. Des trois épreuves, la flexibilité des tiges (contrôle des variables) est celle qui génère le plus haut pourcentage de réponses formelles.

Les moyennes et les écarts types à chaque épreuve et au total pour chaque regroupement de programmes ainsi que pour chaque niveau des variables indépendantes sont présentés au tableau 4. 


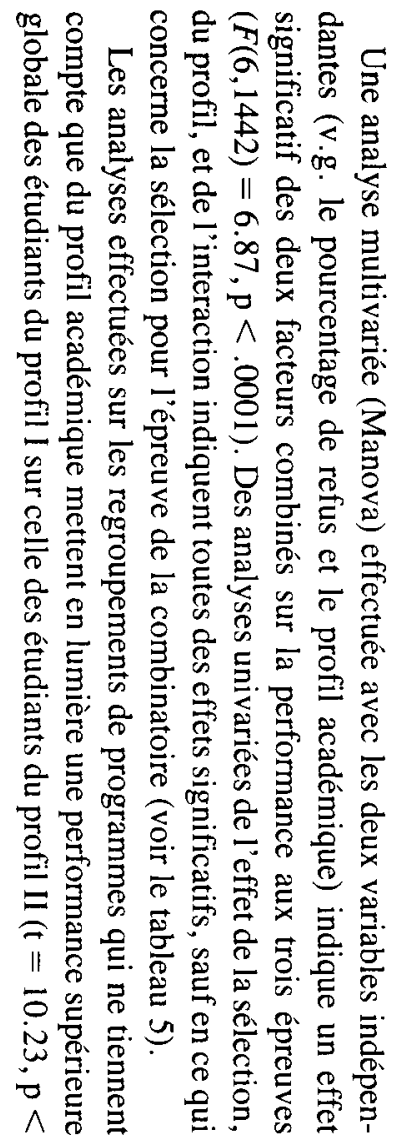

Tableau 3

Moyennes, écarts-type et pourcentages des groupes à léchelle dinformation, aux épreuves du contróle des variables, de la combinatoire, des proportions, et au total.

\begin{tabular}{|c|c|c|c|c|c|}
\hline \multirow[t]{3}{*}{ PROGRAMMES } & INFORMATION & $\begin{array}{r}\text { CONTROLE DES } \\
\text { VARIABLES }\end{array}$ & COMBINATOIRE & PROPORTIONS & TOTAL \\
\hline & $\left(N^{D}=10\right)$ & $(N=9)$ & $(N=15)$ & $(N=8)$ & $(N=32)$ \\
\hline & MOY (E,L)\% & MQY,SE,T) \% & MOY (ET) \% & MOY(ET) \% & MOY $(E, T)$ \\
\hline B.A.Sa & $8,8(0,9) 88$ & $5,6(1,7) 62$ & $8,5(2,2) 57$ & $4,2(2,0) 53$ & $18,4(3,7) 58$ \\
\hline PSYCHOLOGIE & $9,0(0,9) 90$ & $5,7(1,6) 63$ & $9.2(3,0) 61$ & $4,7(2,2) 58$ & $19.7(5,0) 61$ \\
\hline BIOLOGIE & $9,3(0,9) 93$ & $6,4(1,2) 68$ & $9,3(2,6) 60$ & $4,8(2,2) 62$ & $20.6(4.2) 63$ \\
\hline DROIT & $8,9(1,1) 89$ & $5,8(1,9) 64$ & $8,4(2,4) 56$ & $4,7(2,1) 58$ & $19,0(4,8) 59$ \\
\hline GENIE & $9,5(0,5) 95$ & $6,8(1,0) 75$ & $10,7(2,3) 71$ & $7,8(0,4) 97$ & $25,4(2,8) 78$ \\
\hline PHILOSOPHIE & $9,0(1,0) 90$ & $5,8(2,1) 64$ & $8,0(3,3) 53$ & $4,5(2,5) 48$ & $19.6(5.9) 55$ \\
\hline INFORMATIQUE & $9,4(0,8) 94$ & $6,7(1,3) 73$ & $9,7(2,7) 65$ & $5.6(1,9) 67$ & $22,0(3,5) 68$ \\
\hline B.E.P.P. & $9,2(0,8) 92$ & $6,1(1,4) 67$ & $8,4(2,5) 56$ & $4,7(2,1) 55$ & $19.3(3.7) 59$ \\
\hline ADMINISTRATION & $9,1(1,0) 91$ & $6,4(1,5) 71$ & $8,7(2,6) 62$ & $5,6(2,1) 76$ & $20.7(4.1) 68$ \\
\hline MEDECINE & $8,9(1,0) 89$ & $7,6(1,3) 84$ & $10,5(1,7) 70$ & $6,0(2,3) 76$ & $24,3(3,3) 75$ \\
\hline SC.INF ${ }^{a}$ & $8,5(0,9) 85$ & $5,9(1,7) 68$ & $8.1(1.7) 55$ & $4,1(2,2) 53$ & $18,3(3,6) 59$ \\
\hline PSED ${ }^{a}$ & $9,1(1,0) 91$ & $6,4(1,7) 70$ & $9,2(2,2) 60$ & $5,1(2,3) 65$ & $20,8(4,3) 64$ \\
\hline TOTAL & $9.1(0.9) 91$ & $6,2(1,6) 68$ & $9,0(2,6) 60$ & $5,1(2,2) 62$ & $20,4(4,4) 63$ \\
\hline
\end{tabular}

B.A.S: $\quad$ Baccalauréat en adaptation scolalre

B.E.P.: Baccalaureat en enseignement pré-scolatre et primaire

SC.INF.: Sciences infirmières

PS.E.D.: PSycho-éducation

$D_{N}=$ le nombre d'items 


\section{TABLEAU 4}

Moyennes et écarts-type aux épreuves du contrôle des variables (CVR), de la combinatoire (CBR), des proportions (PRT) et au total (TOT) en fonction du pourcentage de refus, du profil académique et des regroupements

\begin{tabular}{|c|c|c|c|c|}
\hline \multicolumn{5}{|l|}{ Profil } \\
\hline \multirow[t]{3}{*}{ Académique ${ }^{a}$} & \multirow[t]{3}{*}{ Schèmes } & \multicolumn{2}{|c|}{$\%$ de refus } & \multirow[t]{2}{*}{ Total } \\
\hline & & $>42 \%$ & $<15 \%$ & \\
\hline & & MOY (E.T) & MOY. (E,T) & MOY (E.T.) \\
\hline \multirow{5}{*}{$\mathbf{I}$} & & GROUPEA & GROUPEB & \\
\hline & CVR $(9)^{b}$ & $7.16(1.21)$ & $6.41(1.21)$ & $6.77(1.26)$ \\
\hline & CBT (15) & $10.70(2.18)$ & $9.39(2.66)$ & $10.01(1.52)$ \\
\hline & PRT (8) & $7.20(1.66)$ & $4.81(2.29)$ & $5.97(2.34)$ \\
\hline & TOT (32) & $25.07(3.04)$ & $20.59(4.23)$ & $22.75(4.32)$ \\
\hline \multirow{6}{*}{ II } & & GROUPEC & GROUPED & \\
\hline & CVR (9) & $6.44(1.51)$ & $6.70(1.36)$ & $6.54(1.46)$ \\
\hline & CBT (15) & $8.70(2.61)$ & $9.70(2.65)$ & $9.09 \quad(2.66)$ \\
\hline & PRT (8) & $5.61(2.11)$ & $5.61(1.93)$ & $5.61(2.03)$ \\
\hline & TOT (32) & $20.77(4.16)$ & $22.02(3.59)$ & $21.25(3.99)$ \\
\hline & & GROUPEE & GROUPEF & \\
\hline \multirow{4}{*}{ III } & CVR (9) & $6.16(1.71)$ & $5.79(1.79)$ & $5.99(1.75)$ \\
\hline & CBT (15) & $8.85(2.46)$ & $8.33(2.33)$ & $8.62(2.41)$ \\
\hline & PRT (8) & $4.93(2.22)$ & $4.23(2.19)$ & $4.63(2.23)$ \\
\hline & TOT (32) & $19.96(4.41)$ & $18.56(4.12)$ & $19.35(4.33)$ \\
\hline \multirow{4}{*}{ TOTAL } & CVR (9) & $6.40(1.62)$ & $6.12(1.63)$ & $(1.6)$ \\
\hline & CBT (15) & $9.11(2.55)$ & $8.85(2.54)$ & $(2.6)$ \\
\hline & PRT (8) & $5.49 \quad(2.25)$ & $4.66 \quad(2.22)$ & $(2.2)$ \\
\hline & TOT (32) & $21.01(4.52)$ & $19.75(4.27)$ & $(4.4)$ \\
\hline
\end{tabular}

a I: Sciences pures et appliquées

II: mathématiques

111: sciences sociales et humaines

b Le contenu des ( ) indique le nombre d'items

$.01)$ et du profil III $(t=64.04, p<.0001)$. Les étudiants du profil II ont aussi des résultats supérieurs à ceux des sujets du profil II $(\mathrm{t}=24.91, \mathrm{p}<.0001)$.

Par contre, des différences significatives au plan de la performance globale sont observables entre les divers programmes regroupés à l'intérieur même du profil I $(\mathrm{f}(2,136)=26.11, \mathrm{p}<.0001)$, du profil II $(\mathrm{t}=4.24, \mathrm{p}<.05)$ et du profil III $(\mathrm{f}(6,416)=3.50, \mathrm{p}<.002)$.

Un test de Scheffé effectué sur les résultats obtenus par les six groupes formés en fonction des deux critères de regroupement indique que le groupe $\mathrm{A}$, caractérisé par un contenu scientifique et mathématique, ainsi que par une sélection serrée à 


\section{TABLEAU 5}

Analyses univariées de l'effet de la sélection, du profil et de l'interaction sur la performance aux épreuves du contrôle des variables, de la combinatoire, des proportions, et du total.

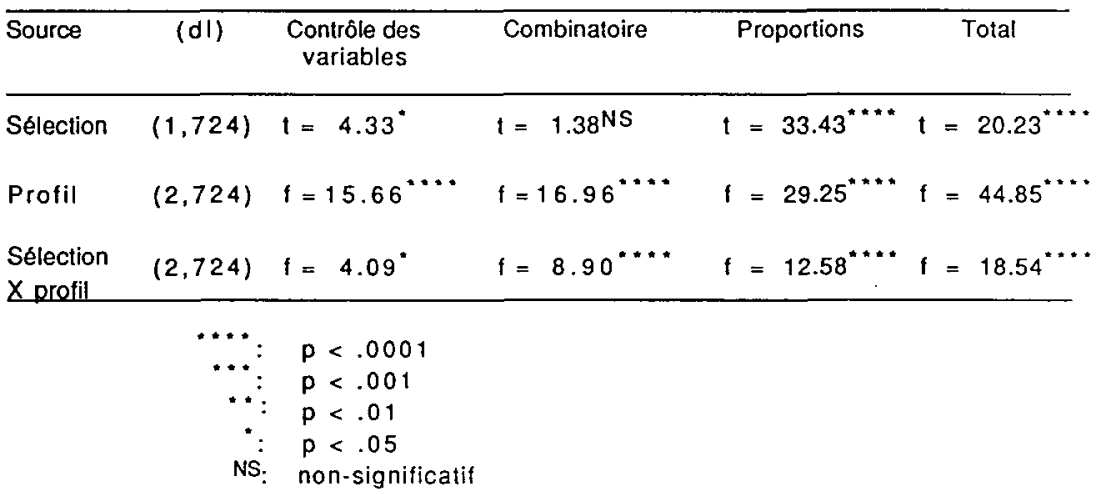

l'entrée, présente une performance supérieure à celle de tous les autres groupes aux épreuves de proportions et au total (voir le tableau 6). À l'opposé, les résultats du groupe $\mathrm{F}$, caractérisé par l'absence de contenu scientifique ou mathématique ainsi que par une sélection très peu discriminante, s'avèrent significativement plus faibles que ceux de tous les autres groupes au total. Des différences significatives au plan des résultats obtenus aux autres épreuves sont aussi apparentes entre les deux groupes extrêmes ( $A$ et $F$ ) et les groupes $B, C, D$ et $E$. Ces différences varient d'un groupe à l'autre : le groupe $\mathrm{A}$ ne se démarque pas des groupes $\mathrm{B}, \mathrm{C}$ et $\mathrm{D}$ à l'épreuve du contrôle des variables, et présente une performance équivalente à celle du groupe $\mathrm{D}$ à l'épreuve de la combinatoire. Les résultats du groupe $\mathrm{F}$ sont plus faibles que ceux des groupes $\mathrm{A}$ et $\mathrm{D}$ pour toutes les épreuves, et inférieurs à ceux du groupe $B$ à l'épreuve de combinatoire, à ceux du groupe $C$ au contrôle des variables et aux proportions et enfin à ceux du groupe $\mathrm{E}$ aux proportions.

Les groupes B, C, D et E, dont la performance est favorisée ou handicapée par un seul facteur (le profil ou la sélection) présentent une performance similaire sur tous les points, sauf le groupe $\mathrm{D}$ qui obtient des résultats légèrement supérieurs à ceux du groupe $\mathrm{E}$ au total.

Pour les profils I et III une sélection plus serrée se traduit par de meilleurs résultats. Les groupes du profil II (C et D) obtiennent des résultats différents de ceux générés par le test de Scheffé; les étudiants d'informatique présentent une performance supérieure à celle des étudiants d'administration à l'épreuve de la combinatoire $(\mathrm{t}=6.04, \mathrm{p}<.05)$ et au total $(\mathrm{t}=4.24, \mathrm{p}<.05)$, et ce bien que la sélection soit plus rigoureuse pour ces derniers (voir la figure 1).

À l'intérieur de chacune des trois cellules constituées par regroupement, (A, E et F), il n'y a pas de différence significative de la performance globale des étudiants provenant des divers programmes agrégés, que ce soit pour le groupe $\mathrm{A}$ $(t=2.26, N . S$. $)$, le groupe $E(f(3,229)=2.60$, N.S. $)$, ou le groupe $F(f(2,178)=$ 
Différences significatives au test de Scheffé entre les

6 groupes aux épreuves du contrôle des variables

(CVR), de la combinatoire (CBT), des proportions (PRT) et au total (TOT).

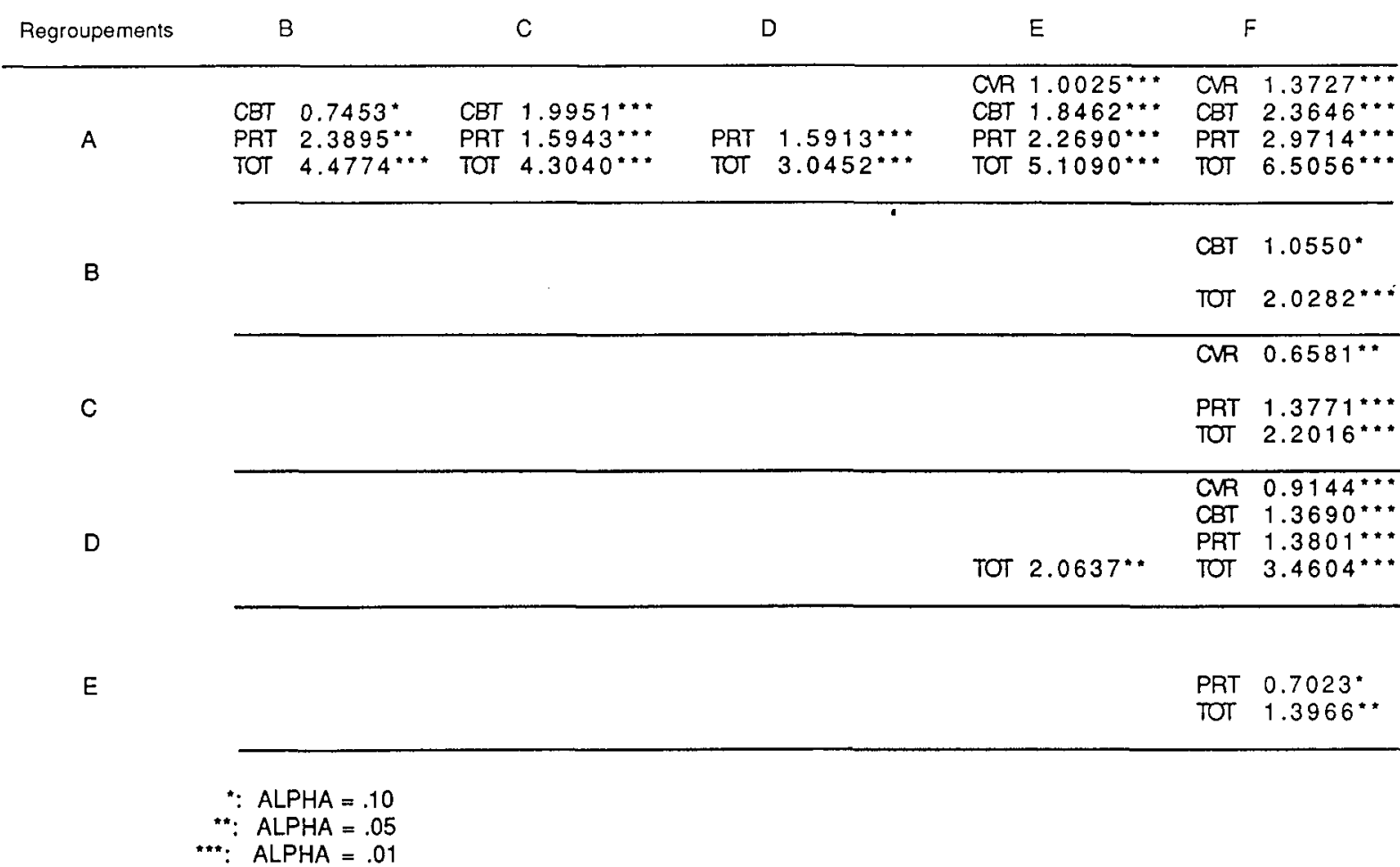




\section{FIGURE 1}

Variation des pourcentages de réussite à l'épreuve de la combinatoire et à l'ensemble des items formels en fonction du profil et de la sélection

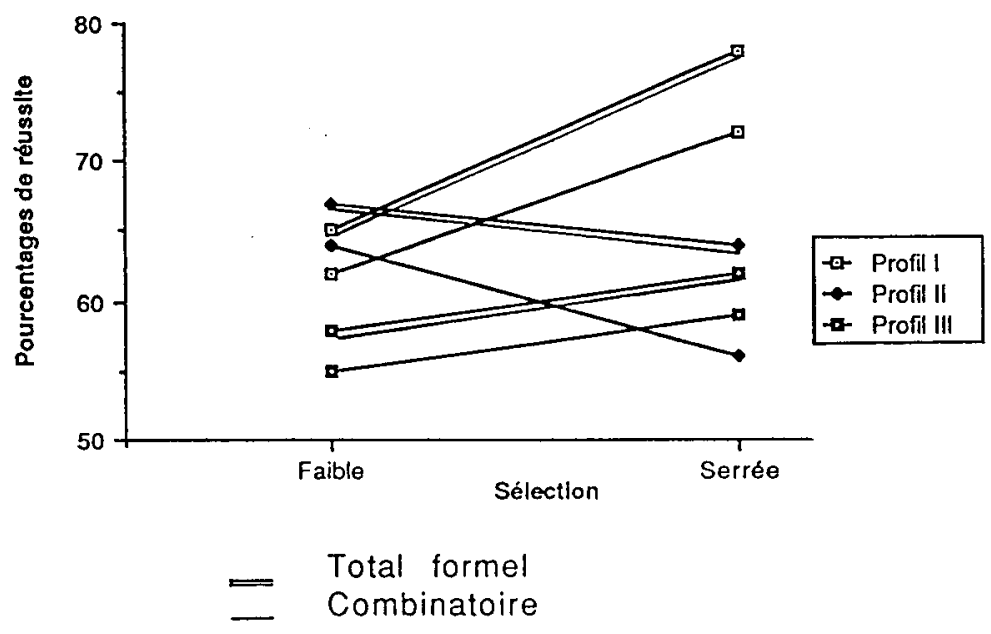

1.00, N.S.). Cependant, au sein du groupe $A$, les étudiants du programme de génie présentent une performance supérieure aux épreuves de proportions $(t=22.70, \mathrm{p}$ $<.0001$ ), alors que les étudiants de médecine les surclassent à l'épreuve du contrôle des variables $(t=6.99, p<.05)$. Le groupe $A$ est cependant homogène eu égard à la performance à l'épreuve de la combinatoire $(t=0.11$, N.S.) et au total, tel que vu plus haut.

\section{DISCUSSION}

Seront discutés ici : l'impact de la sélection et du profil sur la performance cognitive, les erreurs générées par l'utilisation d'un seul critère de regroupement, l'antériorité de la sélection sur la familiarité avec le contenu et enfin l'opportunité différentielle d'utiliser à profit les opérations formelles en fonction des programmes.

Les résultats obtenus ici mettent en évidence un impact significatif du profil, de la rigueur de la sélection (sauf à l'épreuve de la combinatoire), et de l'interaction entre ces deux facteurs, sur la performance cognitive. Les données du test de Scheffé peuvent être synthétisées comme suit: le groupe A obtient les meilleurs résultats, les groupes $\mathrm{B}, \mathrm{C}, \mathrm{D}$ et $\mathrm{E}$ présentent une performance relativement homogène, mais globalement plus faible, alors que le groupe $\mathrm{F}$ est significativement plus faible que tous les autres. Tout se passe comme si les deux variables indépendantes retenues, le profil et la sélection, avaient un effet maximal lorsque leur influence est convergente, une influence divergente se traduisant par la 
compensation d'un effet par l'autre. Ainsi, la performance la plus forte est associée à une conjonction de sélection rigoureuse et de contenu à caractère scientifique, alors que les résultats les plus faibles proviennent du groupe chez qui ces deux paramètres sont inversés. Les analyses effectuées montrent que chacun des trois niveaux de la variable profil est associé à une performance différentielle elle-même affectée à la hausse ou à la baisse par la sélection à l'entrée, sauf en ce qui a trait aux étudiants du profil II. Ces derniers obtiennent globalement des résultats qui confirment l'effet de la familiarité avec le contenu, leur performance étant supérieure à celle des étudiants du profil III et inférieure à celle des sujets du profil I, en relation avec une exposition différentielle aux contenus logicomathématiques. On peut donc s'attendre à ce que l'effet de la sélection se traduise par une performance supérieure du groupe $\mathrm{C}$ (administration) en comparaison avec le groupe D (informatique), de même profil. Cependant, la sélection n'a pas produit l'effet anticipé ici. Les étudiants d'informatique obtiennent des résultats supérieurs à l'épreuve de la combinatoire et au total qui contribuent à diminuer la variance provoquée par la sélection au point de rendre son effet non significatif pour le schème de la combinatoire. Comment expliquer ces résultats contradictoires? Bien que la structure d'accueil requise au niveau des études préuniversitaires soit la même pour les deux groupes, il faut considérer que les activités académiques auxquelles ces étudiants participent dans leurs programmes respectifs sont fort différentes. Ainsi, les étudiants d'informatique sont inscrits en première année dans des cours obligatoires et optionnels dont le contenu est presque exclusivement de nature logico-mathématique (v.g. programmation, télématique, algorithmique, modèles statistiques et mathématiques etc.), alors que les étudiants d'administration sont soumis à un régime d'études où la composante principale relève de la psychologie et du marketing (v.g. principes d'administration, aspects humains de l'organisation, gestion du personnel etc.) Il est donc possible que la variable principale dans ce cas précis ne soit pas la sélection, ni le profil antérieur, mais le contenu auquel les étudiants sont actuellement exposés et qu'à ce titre, les étudiants d'informatique sont peut-être les seuls à relever authentiquement du profil II. Pour leur part, les étudiants d'administration semblent confrontés à des contenus plus typiques de ceux rencontrés dans le profil III. La performance équivalente des étudiants de ce groupe et des étudiants du groupe E (profil III et sélection serrée) va dans ce sens. Il faut par contre souligner que le test de Scheffé génère des données très conservatrices où l'erreur de type $\mathrm{A}$ est bien contrôlée, ce qui risque d'occulter certaines différences pourtant significatives lorsque l'analyse de variance est utilisée.

Les analyses effectuées indiquent qu'un regroupement de sujets fait uniquement sur la base du profil a comme conséquence d'introduire un biais important, en raison de l'hétérogénéité des performances des sujets issus de programmes comparables sur le plan du profil académique, mais différents en termes de sélection. Ainsi, la performance globale d'un groupe constitué de sujets provenant de programmes ayant un profil similaire peut être affectée à la hausse ou à la baisse en fonction de la présence de sujets ayant fait l'objet d'un tamisage plus ou moins 
important, comme c'est le cas pour les étudiants de biologie qui font partie du profil I mais dont la performance est nettement plus faible que celle des étudiants de médecine et de génie, ou encore pour les étudiants d'adaptation scolaire, qui présentent une performance inférieure à celle de leurs collègues de psychoéducation pourtant du même profil (III). L'utilisation de deux critères de regroupement, le profil et la sélection, a donc permis la création de nouveaux groupes expérimentaux plus homogènes que ceux obtenus par l'application du seul critère du profil académique.

Bien que l'effet du profil académique semble aussi important que celui de la sélection à l'entrée, la familiarité avec le contenu ne peut être considérée d'emblée comme le facteur central dans le cadre de cette recherche. En effet, le tri effectué à l'université n'est que l'aboutissement d'un processus de sélection beaucoup plus global et qui débute dès la fin des études primaires. Les élèves qui présentent les meilleurs résultats suivent une trajectoire scolaire différente de celle de leurs collègues moins doués; l'accès aux programmes à teneur scientifique leur est à toutes fins pratiques réservé, et ce dès l'adolescence, alors que les autres étudiants n'accèdent facilement qu'aux programmes de sciences humaines et sociales. En fait, les programmes universitaires scientifiques, de par leurs exigences en termes d'acquis préalables et de structure d'accueil, reçoivent donc des candidats ayant déjà fait l'objet d'un premier tamisage à l'école secondaire et d'un deuxième, au niveau collégial. Lorsqu'une troisième sélection serrée a lieu lors de l'admission à l'université, il est évident que les étudiants finalement admis se démarqueront de façon assez nette de leurs pairs non soumis au même processus. Les résultats des étudiants de médecine et de biologie sont à cet égard éloquents : la performance cognitive de ces derniers est significativement plus faible. Peut-être faut-il rappeler qu'une demande d'admission en biologie n'est souvent que le deuxième choix des candidats refusés en médecine.

Le contenu de la plupart des programmes scientifiques offre d'emblée aux étudiants la possibilité de vérifier de façon empirique la justesse de leurs hypothèses sur du matériel concret ou quantifiable, ce qui est rarement le cas en sciences humaines et sociales. Certains schèmes pourraient aussi être plus sollicités dans certains programmes, et moins ailleurs. En ce sens, le pourcentage de réussite le plus élevé à l'épreuve du contrôle des variables ( $84 \%$ ) a été obtenu par les étudiants de médecine, pour lesquels la dissociation des facteurs est centrale dans un processus de diagnostic, alors que la performance de groupe la plus forte enregistrée aux deux épreuves de proportions ( $97 \%$ ) a été réalisée par les étudiants de génie, pour lesquels ce schème est essentiel dans nombre de travaux pratiques. Une sélection plus serrée en sciences humaines et sociales permet peut-être d'éliminer les étudiants susceptibles d'avoir une performance moindre et qui ne peuvent compenser l'absence de contenu scientifique et de manipulations concrètes par une capacité supérieure de dissocier la forme du contenu, mais l'aire de vérification de ces programmes (i.e. le contenu et les variables sur lesquels les vérifications doivent être effectuées) est relativement floue et leur contenu s'accompagne souvent de prescriptions morales et de résonnances émotives qui 
s'accommodent mal d'opérations à caractère logico-mathématique. A prime abord, les activités cognitives typiques des sciences exactes peuvent sembler plus exigeantes, mais l'évolution spectaculaire des connaissances dans ce domaine, par opposition aux balbutiements de la sociologie et de la psychologie, entre autres, favorisent une autre interprétation. Les problèmes épistémologiques fort différents rencontrés en sciences exactes et en sciences humaines et sociales témoignent plus d'une disproportion importante au niveau de l'ampleur des efforts à déployer pour mener à terme une entreprise de connaissances lorsque celle-ci se fait dans des univers de contenu si divergents en termes de variables à contrôler et de possibilités de vérification empirique. Dans cette perspective, les échecs rencontrés dépendent peut-être autant des difficultés de l'entreprise que des capacités cognitives des entrepreneurs.

\section{Conclusion}

Le profil académique n'explique pas à lui seul les variations de performance rapportées ici. Les différences significatives induites par la sélection des candidats à l'entrée viennent nuancer quelque peu l'importance accordée à l'adaptation du contenu des épreuves au domaine de spécialisation. Ceci ne diminue en rien l'importance de mieux cerner quelles habiletés cognitives spécifiques sont requises en sciences humaines et sociales, de même que les épreuves les plus susceptibles de générer une performance qui se rapproche plus de la compétence réelle. Les résultats obtenus ici s'incrivent dans la foulée d'autres travaux récents (Lehman, Lemper et Nisbett, 1988) qui mettent aussi en évidence un impact du contenu spécifique de divers programmes universitaires sur le raisonnement. Cependant, cet impact est sans doute exacerbé ou inhibé par la plus ou moins grande rigueur de la sélection des candidats à l'entrée.

Peut-être faut-il questionner ici la théorie piagétienne orthodoxe qui survalorise les opérations logico-mathématiques au détriment d'activités cognitives tout aussi sophistiquées mais plus difficilement identifiables, telles que la métacognition à l'âge adulte (Demetriou et Efklides, 1985; Lefebvre-Pinard, 1980) ou encore l'adaptation des outils cognitifs formels à certaines réalités quotidiennes réfractaires à une saisie uniquement cognitive et logique (Labouvie-Vief, 1977). Ces dernières pourraient être fréquemment requises en sciences humaines et sociales, et ce sans que leur présence puisse être détectée par les épreuves opératoires traditionnelles.

Au delà des aspects théoriques, les résultats présentés ici remettent en cause la légitimité de la sélection telle que pratiquée actuellement à l'université. Le faible tamisage des étudiants des programmes de sciences humaines et sociales permet l'entrée d'un plus grand nombre de candidats à l'université. Cette démocratisation apparente cache cependant le fait que ce sont ces derniers qui contribuent indirectement à financer, par leur nombre, les programmes scientifiques traditionnels qui sont généralement plus valorisés, mieux subventionnés et beaucoup moins accessibles. 
L'acceptation de candidats moins performants dans certains programmes présente des avantages financiers intéressants à court terme. Par contre, à plus longue échéance, cette politique de "portes ouvertes" risque de taxer lourdement les programmes les moins sélectifs, comme c'est fréquemment le cas en sciences humaines et sociales. Il devient donc impératif d'offrir un support et un encadrement particuliers aux étudiants plus faibles, afin d'éviter qu'un accès plus facile à l'université ne se traduise par une dégradation progressive de la qualité de l'enseignement due au nivellement par le bas.

\section{RÉFÉRENCES}

Albanese, M., Brooks, D.W. (1976). Piagetian criteria as predictors in first year courses. Journal of Chemical Education, 53(9), 571-572.

Alcock, J.E., Moore, T.E. (1985). The vulnerability of belief in the conservation of area. Canadian Journal of Behavioral Science, 17(3), 276-283.

Bart, W.M. (1971). The effect of interest on horizontal decalage at the stage of formal operations. The Journal of Psychology, 78, 141-150.

Commons, M.L., Miller, P.M., Kuhn, D. (1982). The relation between formal operational reasoning and academic course selection and performance among college freshman and sophomores. Journal of Applied Developmental Psychology, 3(1), 1-10.

De Garcer, I., Gabel, D.L., Staver, J.R. (1978). Implications of Piagetian Research for higher school research teaching : a review of literature. Science Education, 62(4), 571-582.

DeLisi, R., Staudt, J. (1980). Individual differences in college students' performance on formal operations tasks. Journal of Applied Developmental Psychology, 1, 201-208.

Demetriou, A., Efklides, A. (1985). Structure and Sequence of formal and post formal thought: General patterns and individual differences. Child Development, 56, 1062-1091.

Dunbar, A.M., Taylor, B.W. (1983). Investigation of college students academic achievement based on their piagetian cognitive level and American College Test scores. Psychological Reports, $53,923-930$.

El-Gosbi, A.M. (1982). A study of the understanding of science processes in relation to Piaget's cognitive development at the formal level, and other variables among prospective teachers and college science majors. Dissertation Abstracts International, 43(6-A), 1914.

Enwieme, A.A.E. (1976). The incidence of formal operations of students in eight subject areas of the Nustep program at the University of Nebraska. Dissertation Abstracts International, 37A, 2761-2762.

Good, R. (1983). Comments on "College chemistry and Piaget": the relationship of aptitude and achievement measures. Journal of Research in Science Teaching, 20(2), 181-182.

Higele, P. (1978). Étude de la mesure du niveau opératoire des adultes. Psychologie française, 23(2), 95-105.

Inhelder, B., Piaget, J. (1955). De la logique de l'enfant à la logique de l'adolescent. Paris : Presses universitaires de France.

Karplus, R., Karplus, E., Formisano, M., Paulsen, A.C. (1977). A survey of proportional reasoning in seven countries. Journal of Research in Science Teaching, 14(5), 411-417.

Kornbrot, D.E. (1987). Science and psychology degree performance. Bulletin of the British Psychological Society, 40, 409-417.

Kuhn, D., Brannock, J. (1977). Development of the isolation of variables scheme in experimental and "natural experiment" context. Developmental Psychology, 13(1), 9-14.

Labouvie-Vief, G. (1977). Adult cognitive development: in search of alternative interpretations. Merril-Palmer Quarterly, 13, 227-263. 
Larivée, S., Cormier, P. (1982). Le schème du contrôle des variables, un instrument du raisonnement scientifique. 1. Sa nature. Spectre, $12(1), 12-16$.

Larivée, S., Normandeau, S. (1985). Maîtrise du schème de la combinatoire (permutations) chez les adolescents en classes spéciales. Canadian Journal of Education, 10 (4), 345-361.

Larivée, S., Normandeau, S. (1984). Performance cognitive de futurs professionnels de l'intervention. The Canadian Journal of Higher Education, 14(2), 33-45.

Larivée, S., Pelletier, D., Gagnon, C. (1986). Tests papier- crayon et mesure des opérations formelles : une revue critique. Revue de psychologie appliquée, 36(2), 151-180.

Larivée, S., Roulin, J.L. (1985). Le schème des proportions : approche développementale. Rapport de recherche no. 32, ERISH, Université de Montréal, EMSA.

Lefebvre-Pinard, M. (1980). Existe-t-il des changements cognitifs chez l'adulte? Revue québécoise de psychologie, I (2), 58-69.

Lehman, D.R. Lempert, R.O., Nisbett, R.E. (1988). The effects of graduate training on reasoning: Formal discipline and thinking about everyday-life events. American Psychologist, 43(6), 431-442.

McKinnon, J.W., Renner, J.W. (1971). Are colleges concerned with intellectual development? American Journal of Physics, 39, 5-9.

Neimark, E.D. (1979). Current status of formal operations research. Human Development, 22, 60-67.

Neimark, E.D. (1978). On the measurement of formal operations. The Formal Operator, 1(3), 10-11.

Parete, J.D. (1978), Formal reasoning abilities of College Age Students: An investigation of the concrete and formal reasoning stages formulated by Jean Piaget. Dissertation Abstracts International, 39(10-A), 6006.

Pelletier, D., Larivée, S., Gagnon, C. (1985). Étude comparative de l'équivalence des tests papier-crayon et de la méthode clinique dans l'évaluation de la pensée formelle. Cahiers de la société Alfred Binet et Théodore Simon, 605(4), 25-42.

Piaget, J. (1970). L'évolution intellectuelle entre l'adolescence et l'âge adulte. Milan : Remise des prix Foneme.

Piaget, J., Inhelder, B. (1963). Les opérations propositionelles ou formelles in P. Fraisse, J. Piaget (Eds). Traité de psychologie expérimentale, VII, (pp. 154-165). Paris : Presses Universitaires de France.

Pulos, S., Linn, M.C. (1981). Generality of the controlling variables scheme in early adolescence. Journal of Early Adolescence, I(1), 26-37.

Staver, J.R. (1982). The Piagetian Logical Operations Test : A group assessment method for measuring formal reasoning patterns. School Science and Mathematics, 82(2), 169-173.

Staver, J.R., Gabel, D.L. (1979). The developmental and construct validation of a group administered test of formal thought. Journal of Research in Science Teaching, 16(6), 535-544.

Torkia-Lagacé, M. (1981). La pensée formelle chez les étudiants de Collège I : Objectif ou réalité? Limoilou : Cegep de Limoilou.

White, K.M., Ferstenberg, A. (1978). Professional specialization and formal operations : The balance task. The Journal of Genetic Psychology, 133, 97-104. 